\title{
Electronic and optical properties of beryllium chalcogenides/silicon heterostructures
}

\author{
Titus Sandu* \\ Département de chimie \\ Université de Montréal \\ C.P. 6128, succursale Centre-ville, \\ Montréal, Québec H3C 3J\%, Canada \\ W. P. Kirk \\ University of Texas at Arlington \\ NanoFAB Center \\ Arlington, Texas 76019
}

(Dated: November 14, 2018)

\begin{abstract}
We have calculated electronic and optical properties of $\mathrm{Si} / \mathrm{BeSe}_{0.41} \mathrm{Te}_{0.59}$ heterostructures by a semiempirical $s p^{3} s^{*}$ tight-binding method. Tight-binding parameters and band bowing of $\mathrm{BeSe}_{0.41} \mathrm{Te}_{0.59}$ are considered through a recent model for highly mismatched semiconductor alloys. The band bowing and the measurements of conduction band offset lead to a type II heterostucture for $\mathrm{Si} / \mathrm{BeSe}_{0.41} \mathrm{Te}_{0.59}$ with conduction band minimum in the Si layer and valence band maximum in the $\mathrm{BeSe}_{0.41} \mathrm{Te}_{0.59}$ layer. The electronic structure and optical properties of various $\left(\mathrm{Si}_{2}\right)_{n} /\left(\mathrm{BeSe}_{0.41} \mathrm{Te}_{0.59}\right)_{m}$ [001] superlattices have been considered. Two bands of interface states were found within the bandgap of bulk Si. Our calculations indicate that the optical edges are below the fundamental bandgap of bulk $\mathrm{Si}$ and the transitions are optically allowed.
\end{abstract}

PACS numbers: 73.21.Cd,73.21.Fg,78.67.De 


\section{INTRODUCTION}

Silicon completely dominates the present semiconductor market with the greatest shares of all sales. It has an ideal bandgap $(1.12 \mathrm{eV})$ for room temperature operation and its oxide $\left(\mathrm{SiO}_{2}\right)$ provides the necessary flexibility to fabricate millions of devices on a single chip. High integration implies high-speed operation that is limited by the interconnect propagation delay of the signal between devices. This constraint suggests that the integration of $\mathrm{Si}$ micro-electronics might be aided by optical interconnection. Unfortunately, silicon does not respond strongly to optical excitations because it is an indirect bandgap semiconductor: the band extrema for electrons and holes are located at different points in the Brillouin zone (Fig. 1). Therefore, intrinsic formation or recombination of electron-hole pairs becomes a three-particle event, which is weaker than a two-body process. Therefore, there are a number of areas where Si cannot compete and this has allowed other materials to dominate such as optoelectronics where the indirect bandgap precludes the use of Si to produce efficient light emitting diodes and lasers. If the performance of Si transistors or circuits could be improved by addition of another semiconductor material then numerous new applications could open up. Silicon-germanium is one such material which may be epitaxially grown on silicon wafers and allows one to engineer the bandgap, energy band structure, effective masses, mobilities and numerous other properties while fabricating circuits using conventional Si processing and tools 1.2

There has been a tremendous effort in exploring the ways of breaking the silicon lattice symmetry and mixing different momentum states in order to induce optical gain. 3.4 The radiative efficiency depends on the competition between non-radiative fast processes and relatively slow radiative processes. To optimize the efficiency we have to eliminate nonradiative channels by using high purity materials and increase the oscillator strength of radiative channels. The above criteria also apply to photovoltaics. One modality for improving the optical response is quantum confinement. Confinement of the charge density in quantum wells (QWs) permits the relaxation of the optical selection rules for an interband transition. In addition, the band folding in superlattice (SL) structures will enhance the absorption. Consequently a Si based SL will reduce the symmetry which translates into band folding toward the zone center; and as a result vertical transitions will be available at energies closer to the indirect bandgap. The discovery of visible light emission from porous 


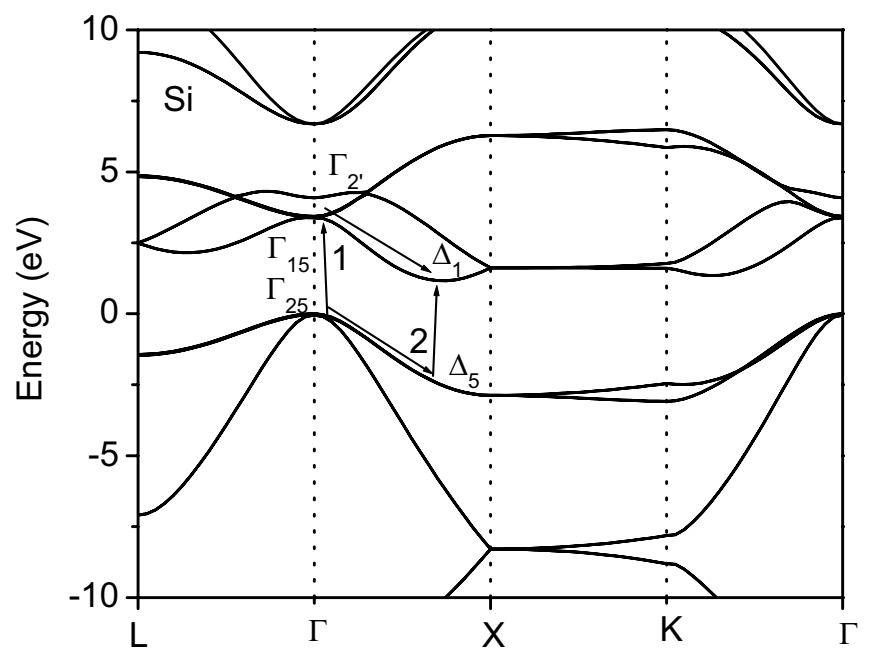

FIG. 1: The band structure of bulk Si with the parameters of $\operatorname{Vogl}$ et al 14 adapted with the spinorbit constant $\Delta=0.045 \mathrm{eV}$. The single group notations are used since the spin-orbit splitting cannot be distinguished on the chosen scale.

silicon has enticed many researchers to reactivate studies of the optical properties of silicon based nanostructures..$^{\frac{5}{2}}$ Efficient visible light emission has been observed in Si-CaF 2 SLs, $\frac{6}{6}$ $\mathrm{Si}_{-} \mathrm{SiO}_{2} \mathrm{SLs}, \frac{7}{2} \mathrm{Si}$ nanopillar structures, $\frac{8}{2}$ and in Si crystallites. $\frac{9}{}$

Beryllium chalcogenides have attracted much attention for the blue wavelength of region light emitting devices or UV detectors. ${ }^{10,11}$ They provide long life-time II-VI lasers because of the strong covalent bonding of Be. BeTe and BeSe are wide-bandgap zinc blende semiconductors with lattice constants on either side of Si: they have the lattice constants of 5.6269 and $5.1477 \AA$, respectively, $3.6 \%$ larger and $5.2 \%$ smaller than Si. Vegard's law indicates that the lattice matched composition with $\mathrm{Si}$ is $\mathrm{BeSe}_{0.41} \mathrm{Te}_{0.59}$. Thus recent developments ${ }^{12.13}$ in the growth of silicon lattice-matched $\mathrm{BeSe}_{0.41} \mathrm{Te}_{0.59}$ open the opportunity for a new class of Si based devices.

In this paper we analyze quantum confinement and band folding in silicon based heterostructures ( $\mathrm{Si} /$ BeSeTe heterostructures) as ways of enhancing light absorption. A semiempirical tight-binding (TB) method ${ }^{15}$ is used throughout the study. Although nowadays ab-initio methods (as they are formulated within density-functional theory) ${ }^{16}$ are becoming routinely utilized for electronic structure and optical properties, they underestimate the bandgaps and therefore they need to be used with care. Moreover, the bandgap problem is solved within the so called GW ( $G=$ Green function, $W=$ screened Coulomb interaction) 
approximation which is a computational demanding task and rarely used for large systems like ours (in our case there is an alloy plus a heterostructure) ${ }^{17}$ In this work we choose a TB method together with a new method ${ }^{18}$ intended to deal with highly mismatched semiconductors, thus considering band-bowing of the BeSeTe alloy. This paper is organized as follows. In section II we discuss methods to improve the light absorption in Si based heterostructures such as quantum confinement in a single (uncoupled) QW and band folding in SL structures. A significant increase in direct absorption is obtained for a narrow uncoupled silicon QW. In this spirit, we calculate the electronic and optical properties of Si/BeSeTe SLs using the TB method mentioned above. In the last section the conclusions are outlined.

\section{IMPROVED OPTICAL ABSORPTION BY QUANTUM CONFINEMENT AND BAND FOLDING}

\section{A. Band Structure of $\mathrm{BeSe}_{0.41} \mathrm{Te}_{0.59}$}

Since both $\mathrm{Si}$ and $\mathrm{BeSe}_{0.41} \mathrm{Te}_{0.59}$ (as we will see below) are indirect band materials, full band calculations are required for a theoretical understanding of electron transport and optical properties in such heterostructures. For this purpose we use the empirical tightbinding (ETB) method as one of the most used tools in research of complex molecular and solid-state systems ${ }^{19}$ Despite the fact that ETB is based on physical approximations such as the one-particle picture, short-range interactions, etc. (a posteriori justified, however), it gives fast and satisfactory results. The short-range nature of the model is suitable for modeling heterointerfaces which are present in such quantum structures. One of the most popular TB models is the $s p^{3} s^{*}$ model.$^{14}$ This is a twenty-band model if spin-orbit coupling is included ${ }^{20}$ The presence of an additional $s^{*}$ orbital is able to reproduce the bandgap in

indirect semiconductors like silicon. The TB parameters of silicon are those from Vogl et al. ${ }^{14}$ augmented with spin-orbit coupling according to Chadi. ${ }^{20}$

BeTe and BeSe are quite new materials in the sense that there are few experimental facts about these semiconductors. The bulk TB parameters of BeTe and BeSe were determined by fitting the GW calculations of Fleszar and Hanke. ${ }^{21}$ The band bowing has been included through a generalization of the band anti-crossing model for highly mismatched semiconductors. ${ }^{18}$ The details of the model and the TB parameters can be found in Ref. 18. 


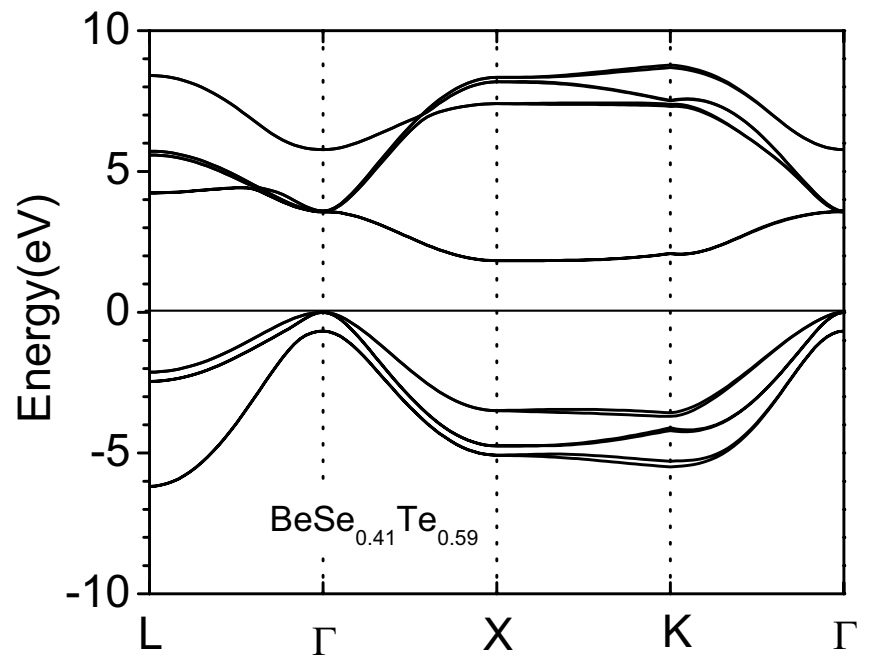

FIG. 2: Energy bands for BeSe $\mathrm{e}_{0.41} \mathrm{Te}_{0.59}$ calculated with TB parameters from Ref. 18 .

The energy band diagram for $\mathrm{BeSe}_{0.41} \mathrm{Te}_{0.59}$ is shown in Fig. 2. The material is seen to be indirect bandgap with the conduction band minimum at the $X$ point. The predicted fundamental bandgap is $1.83 \mathrm{eV}$. The direct bandgap is $3.59 \mathrm{eV}$ and the spin splitting is $0.67 \mathrm{eV}$. Previous electrical measurements ${ }^{12.13}$ indicate a conduction band offset of at most $1.2 \mathrm{eV}$ for the $\mathrm{Si} / \mathrm{BeSe}_{0.41} \mathrm{Te}_{0.59}$ heterostructure. As we will see in the following subsections there are interface subbands induced at the heterostructure interfaces. This fact may overestimate the conduction band offset. Therefore we choose a value of $1 \mathrm{eV}$ for the conduction band offset. This makes the $\mathrm{Si} / \mathrm{BeSe}_{0.41} \mathrm{Te}_{0.59}$ a type II heterostructure. Thus the top of the valence band in $\mathrm{Si}$ is higher than in the top of the valence band in $\mathrm{BeSe}_{0.41} \mathrm{Te}_{0.59}$. The TB parameters and the conduction band offset will be used in the following sections in order to calculate electronic and optical properties of the $\mathrm{Si} / \mathrm{BeSe}_{0.41} \mathrm{Te}_{0.59} \mathrm{SL}$.

\section{B. A Single Quantum Well}

The absorption edges in bulk silicon are indirect and the jumping of the electron from valence band (VB) to conduction band (CB) is assisted by a phonon (in electron transitions from VB to CB the $\boldsymbol{k}$ momentum must be conserved). The band structure of Si is shown schematically in the Fig. 1. The indirect bandgap is $\Delta_{1}-\Gamma_{25}$. The absorption process involves two steps, one is electron-photon interaction and the other is electron-phonon interaction. Basically we have two processes denoted by 1 and 2 . In process 1 an electron is 
first excited to the $\Gamma_{15}$ state and then by an emission of a phonon the electron arrives in the $\Delta_{1}$ CB state. Similarly for process 2 , however, in this process it starts with an emission of a phonon. For this reason, there is a long absorption tail between $1.12 \mathrm{eV}$ and and about 3 $\mathrm{eV}$ that reflects the indirect nature of the bandgap. Then a sharp rise in absorption occurs starting around $3.2 \mathrm{eV}(380 \mathrm{~nm})$ that is associated with the direct transition at $\Gamma$ point $\left(\Gamma_{25}, \rightarrow \Gamma_{15}\right)$ whose energy is $3.4 \mathrm{eV}(365 \mathrm{~nm})$.

In direct bandgap materials light absorption in $2 \mathrm{D}$ systems is formally similar to $3 \mathrm{D}$ systems unlike the light absorption in indirect bandgap systems. The absorption coefficient for an uncoupled $\mathrm{Si} / \mathrm{BeSe}_{0.41} \mathrm{Te}_{0.59} \mathrm{QW}$ has basically three components ${ }^{22}$

$$
\begin{array}{r}
\alpha(\omega)=A\left[\sum_{e h, \lambda_{q}} p_{e h}^{d} \delta\left(E_{g}+E_{e}+E_{h}-\hbar \omega\right)+\sum_{e h, \lambda_{q}} p_{e h}^{a} n_{\lambda_{q}} \delta\left(E_{g}+E_{e}+E_{h}-\hbar \omega-\hbar \omega_{\lambda_{q}}\right)\right. \\
\left.+\sum_{e h, \lambda_{q}} p_{e h}^{e}\left(n_{\lambda q}+1\right) \delta\left(E_{g}+E_{e}+E_{h}-\hbar \omega+\hbar \omega_{\lambda q}\right)\right],
\end{array}
$$

where $A$ is a constant, $p_{e h}^{d}$ gives the direct bandgap contribution and $p_{e h}^{a}$ gives the phonon assisted contribution with 1-phonon absorption, and $p_{e h}^{e}$ is the phonon assisted contribution with 1-phonon emission. Basically,

$$
\begin{gathered}
p_{e h}^{d}=p_{c v} I_{e h}\left(\mathbf{k}_{0}\right) \\
p_{e h, \lambda \boldsymbol{q}}^{e}=p_{e h, \lambda \boldsymbol{q}}^{a}=p_{c v} I_{e h}\left(\mathbf{q}-\mathbf{k}_{0}\right) R_{\lambda} .
\end{gathered}
$$

$p_{c v}$ is the bulk dipole matrix element between bands, $R_{\lambda}$ is the matrix element contribution from electron-phonon interaction, and $I_{e h}$ is the overlap between the envelope-functions at different Brillouin points:

$$
I_{e h}(\mathbf{q})=\int d \mathbf{r} \psi_{e}^{*}(\mathbf{r}) \psi_{h}(\mathbf{r}) e^{-i \mathbf{k}_{0} \mathbf{r}},
$$

where $\mathbf{k}_{0}$ is the location in $k$-space of each conduction valley. For silicon, if $z$ is the growth direction, then the 2 valleys along z-directions (with $k_{y}=k_{x}=0$ ) are responsible for direct transitions. The other four valleys contribute to phonon-assisted absorption. For infinite wells the overlap integral $I_{e h}$ has the behavior depicted in Fig. 3. For very narrow QW's the 


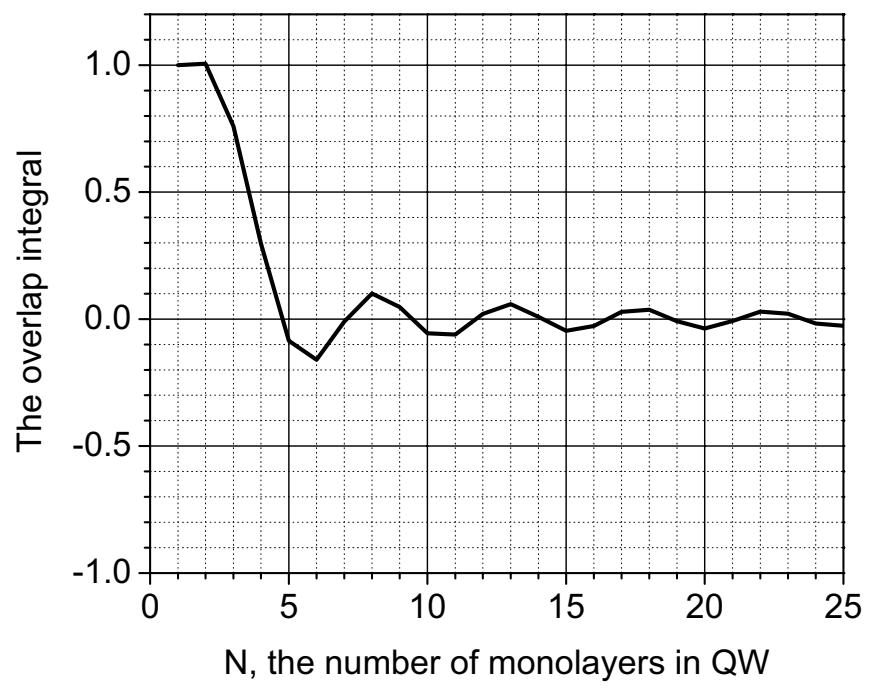

FIG. 3: The overlap integral (Eq.(4) for infinite wells in silicon. The wave vector $\mathbf{k}_{0}$ in Eq.(44) is the wave vector for silicon conduction valleys along $z$-axis.

overlap integral tends to one, which means that in a genuine $2 \mathrm{D}$ system only the transverse momentum has to be conserved. For the other asymptotic limit, i.e. very wide well, the overlap integral vanishes. In physical terms, this says that the system became genuinely $3 \mathrm{D}$ and any electromagnetic transition has to be vertical in the absence of phonons. Moreover, from Fig. 3 we may expect to have a strong direct transition (with an overlap integral no less than 0.1) for a QW with a width up to $20 \AA$, i.e. an ultrathin QW. In the following we will discuss the possibility of improving the oscillator strength in $\mathrm{Si} / \mathrm{BeSe}_{0.41} \mathrm{Te}_{0.59}$ based SLs.

\section{Electronic Structures of $\left(\mathbf{S i}_{2}\right)_{n} /\left(\mathbf{B e S e}_{0.41} \mathbf{T e}_{0.59}\right)_{m}$ [001] Superlattices}

The use of SL structures relaxes the condition of ultrathin QW. The alternation of QWs and barriers along [001] growth axis will generate a band folding in the SL and band mixing of zone-center and zone edge states. ${ }^{23.24}$ The band mixing will inherently enhance the oscillator strength for direct transition in Si structures. On the other hand, band folding will induce states in which vertical transitions are possible at energies far lower than $3.4 \mathrm{eV}$, the lowest energy for vertical transitions in bulk Si.

We consider a $\mathrm{Si} / \mathrm{BeSe}_{0.41} \mathrm{Te}_{0.59} \mathrm{SL}$ whose layers are perpendicular to [001] direction. We employ the nearest neighbor $s p^{3} s^{*}$ TB Hamiltonian including spin-orbit interaction. Al- 

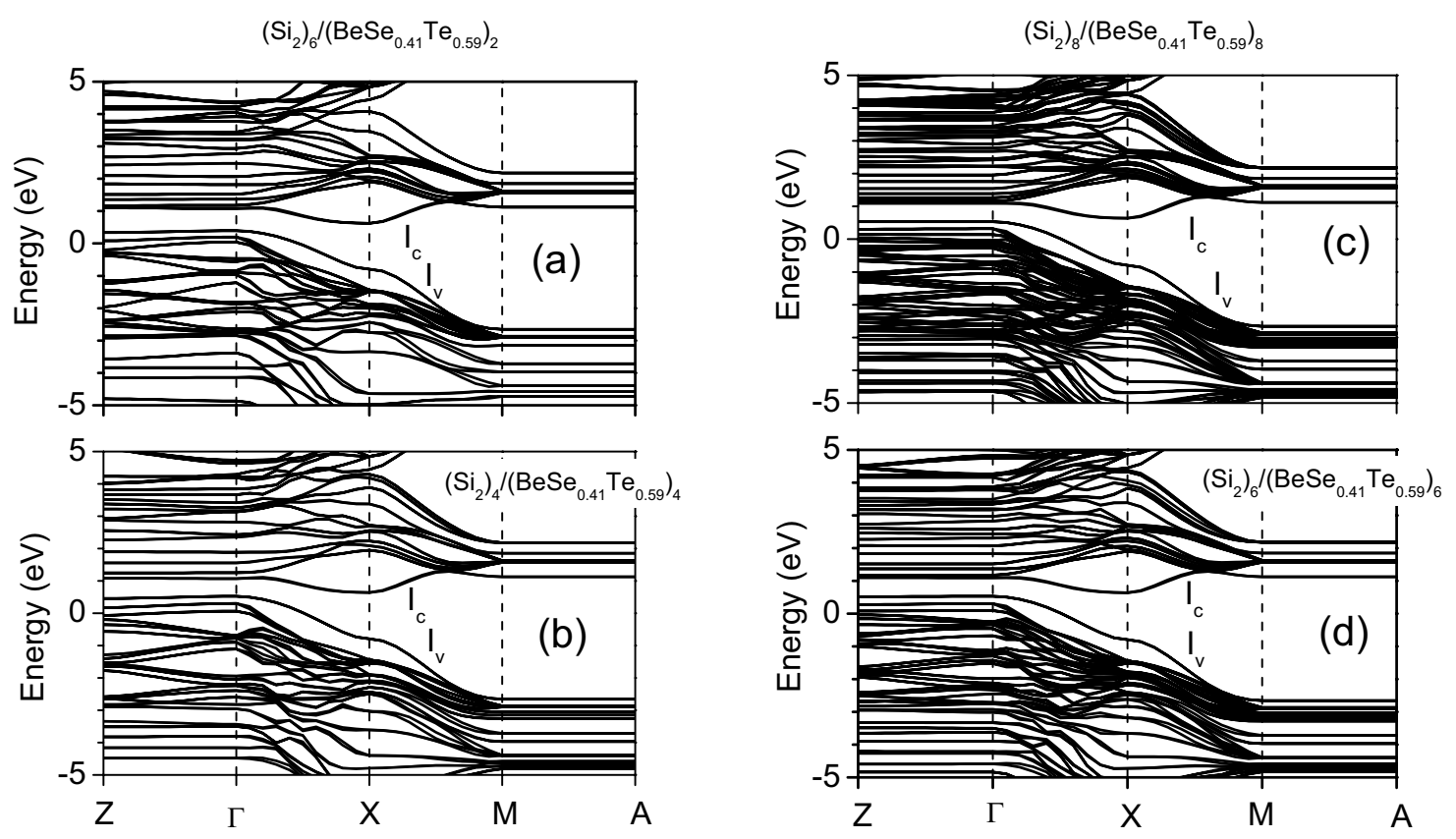

FIG. 4: Band structures of $\left(\mathrm{Si}_{2}\right)_{n} /\left(\mathrm{BeSe}_{0.41} \mathrm{Te}_{0.59}\right)_{m}$ [001] superlattices for (a) $n=6, m=2$, (b) $n=m=4$, (c) $n=m=8$, and (d) $n=m=6$. The interface bands are $\mathrm{I}_{v}$ and $\mathrm{I}_{c}$.

though there are optimized nearest-neighbor TB parameters in the literature ${ }^{25}$ we chose the parameters given in Ref. 14. These parameters include the chemical trends which are needed for an overall description of both valence and conduction bands. As it was noted before, the optimized nearest-neighbor parameters may have unphysical interaction energies and they are dedicated for either valence band or conduction band calculations. ${ }^{25}$ Moreover the nearest-neighbor model has the inability to reproduce the transverse/light X-valley effective mass. However, the conduction band edges in low-dimensional structures will be determined by the longitudinal/heavy effective mass at the conduction band minimum. Therefore we opted for a less accurate yet a very representative set of parameters because these parameters are within the physical capabilities of the nearest neighbor model.

We denote this SL as a $\left(\mathrm{Si}_{2}\right)_{n} /\left(\mathrm{BeSe}_{0.41} \mathrm{Te}_{0.59}\right)_{m}$ SL with $n$ two-atom thick layers of $\mathrm{Si}$ and $m$ two-atom thick layers of $\mathrm{BeSe}_{0.41} \mathrm{Te}_{0.59}$ repeated periodically. The defined supercell consists of $2(n+m)$ adjacently bonded atoms $\mathrm{Si}, \mathrm{Si}, \ldots . . \mathrm{Si}, \mathrm{Be}, \mathrm{Te} / \mathrm{Se}, \mathrm{Be}, \mathrm{Te} / \mathrm{Se}, \ldots . . \mathrm{Be}$, $\mathrm{Te} / \mathrm{Se}$. Covalent bonds between $\mathrm{Si}$ on the one hand and $\mathrm{Be}$ or $\mathrm{Se} / \mathrm{Te}$ on the other are not electrically neutral. ${ }^{26}$ For Si-based heterostructures special care has to be exercised due to excess/deficiency of electrons at interface and due to antiphase domains. ${ }^{27}$ These problems can be overcome by vicinal growth and passivated surfaces: $\stackrel{12,13}{13}$ In this way one can minimize 
the interface charging effects. Moreover, self-consistent ab-initio calculations show that the electric fields due to difference in ionicity between the two semiconductor constituents of a superlattice dominate the total electric field. ${ }^{28}$ Therefore the accumulated charge with respect to the bulk contributes in a much lesser degree to the total electric field in the superlattice structure. In addition to that, the ETB fully self-consistent calculations as those described by Della Sala and coworkers ${ }^{29}$ are not much different from those non-selfconsistent calculations provided that the current densities in the confined system are low ${ }^{30}$ All the above facts lead us to conclude that a "zero-field" approximation will give us at least a qualitative picture of $\mathrm{Si} / \mathrm{BST}$ structures.

The TB matrix elements of the $\mathrm{Si} / \mathrm{BeSe}_{0.41} \mathrm{Te}_{0.59} \mathrm{SL}$ are taken directly from those bulk values. The on-site energies of the $\mathrm{BeSe}_{0.41} \mathrm{Te}_{0.59}$ are accordingly changed to match the valence band offset at the interface. Simple averages were used to supply the parameters connecting different materials at the interface. Since the spin orbit must be included, the SL Hamiltonian will be represented as having $20(n+m)$ functions. Once the TB matrix elements have been established the SL band structure reduces to the diagonalization of the $20(n+m) \times 20(n+m)$ matrix Hamiltonian. The band structure of $\left(\mathrm{Si}_{2}\right)_{n} /\left(\mathrm{BeSe}_{0.41} \mathrm{Te}_{0.59}\right)_{m}$ SL for $m=2$ and $n=6, m=n=4, m=n=8$, and $m=n=6$ are displayed in Fig. 4. The zero of energy corresponds to the top of the valence band in bulk Si. The band folding effect can be seen as many crowded subbands. Two interface bands $\left(\mathrm{I}_{v}\right.$ and $\left.\mathrm{I}_{c}\right)$, one empty and one occupied, were found. They lie in the lower and upper parts of the bandgap of bulk silicon, respectively. The origin of these interface bands rests on the polar nature of the interface as

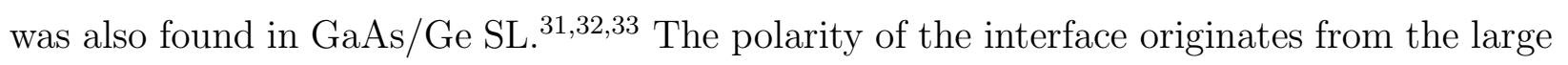
differences in the on-site energies for the constituent atoms ( $\mathrm{Si}$ and Be or Se/Te). Even if a (110) non-polar interface is used, one interface band is still found in II-VI/IV SLs. ${ }^{34.35}$ We calculated the planar charge density of some of the occupied and empty band edge states of the SL with $\mathrm{m}=\mathrm{n}=4$ at the $\Gamma$ point in the Brillouin zone. The planar charge density of some of the band edge states and interface states are depicted in Fig. 15. By interface states we mean states that die away within a few layers from the interface. We denote by $\Gamma^{I v}$, the interface states of the $I_{v}$ band and by $\Gamma^{I c}$, the interface states of the $I_{c}$ band at $\Gamma$ point. The charge density of the $\Gamma^{I v}$ interface state has a maximum at the Be-Si interface, while the next occupied state is more confined in the $\mathrm{BeSe}_{0.41} \mathrm{Te}_{0.59}$ layer, but it still spills over into the Si barrier. The third occupied state spills more over into the Si slab, not only 

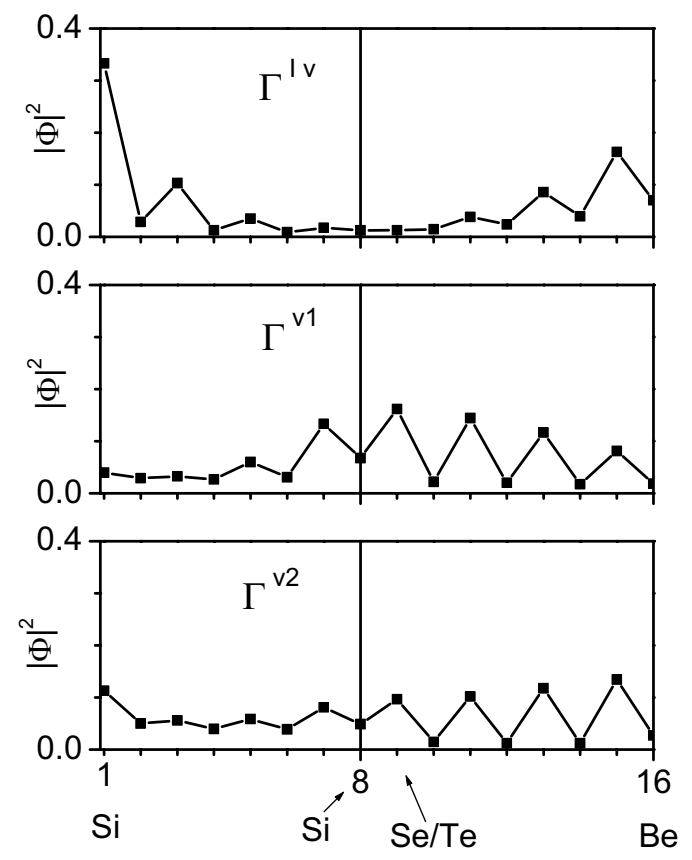
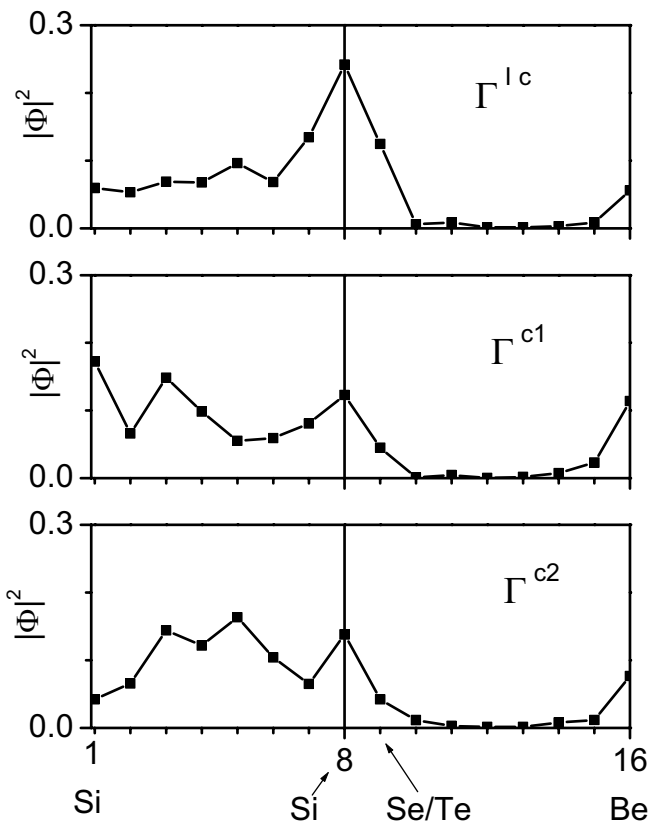

FIG. 5: Planar charge densities in the $\left(\mathrm{Si}_{2}\right)_{4} /\left(\mathrm{BeSe}_{0.41} \mathrm{Te}_{0.59}\right)_{4}$ [001] superlattice at $\Gamma$ point. The occupied states are the interface state $\Gamma^{I_{V}}$ and the first top two confined states $\left(\Gamma^{V 1}\right.$ and $\left.\Gamma^{V 2}\right)$. The empty states are the interface state $\Gamma^{I_{c}}$ and the first two confined states $\left(\Gamma^{C 1}\right.$ and $\left.\Gamma^{C 2}\right)$. The solid vertical lines denote the interfaces.

due to interface but also due to the fact that the state is closer to the top of hole barrier in Si. The charge density of the $\Gamma^{I c}$ interface state has a maximum at the Si-Se/Te interface, while the next empty state is confined, but it is still localized toward the interfaces. Only the third empty state is genuinely confined to the silicon slab. We believe that the charge distribution of the interface subbands is related to the acceptor behavior of Be and donor behavior of Se and Te with respect to $\mathrm{Si}^{36}$

\section{Optical Properties of $\left(\mathbf{S i}_{2}\right)_{n} /\left(\mathbf{B e S e}_{0.41} \mathbf{T e}_{0.59}\right)_{m}$ [001] Superlattices}

The electronic contribution to absorption spectrum is given by $\sigma_{a b s}(\omega) \sim \omega \varepsilon_{2}(\omega)$, where $\sigma_{a b s}(\omega)$ is the absorption coefficient and 19

$$
\varepsilon_{2}(\omega)=\frac{2 \pi^{2} \hbar e^{2}}{m \omega \Omega} \sum_{c, v, k} f_{c v, k} \delta\left(E_{c, k}-E_{v, k}-\hbar \omega\right)
$$

is the imaginary part of the dielectric function. Here $m$ is the electron mass, $\Omega$ is the vol- 
ume, $e$ is the electron charge, $\hbar$ is the Planck constant and $f_{c v, k}$ is the oscillator strength for the direct transition from the state $|v, \mathbf{k}\rangle$ to $|c, \mathbf{k}\rangle$, with the photon momentum neglected. In Eq. (5) it is invoked the electric dipole approximation in the low-temperature case. In ETB the expressions for optical absorption and, in general, the interaction with the electromagnetic field are different than those encountered in conventional quantum mechanics due to incompleteness of the basis $\frac{37}{3}$ In the electric dipole approximation, however, the expressions of optical absorption are the same. The full temperature-dependent equations for optical absorption are derived in Refs. 38 and 39. Beyond the dipole approximation the standard expression (5) is no longer valid. The problem is treated exhaustively in Ref. 40, where it is explicitly shown how the Hamiltonian is altered by the vector potential of the electromagnetic field.

The oscillator strength is defined as

$$
f_{c v, k}=\frac{2}{m} \frac{|\langle c, \mathbf{k}|\boldsymbol{\varepsilon} \cdot \mathbf{p}| v, \mathbf{k}\rangle|^{2}}{E_{c, k}-E_{v, k}} .
$$

In Eq. (6) $|v, \mathbf{k}\rangle$ and $|c, \mathbf{k}\rangle$ are the valence and conduction band eigenstates, $E_{v, k}$ and $E_{c, k}$ are their corresponding energies, $\boldsymbol{\varepsilon}$ is the polarization of light, and $\mathbf{p}$ is the momentum operator. In the empirical tight-binding approach the momentum matrix element is defined as 38

$$
\langle c, \mathbf{k}|\mathbf{p}| c, \mathbf{k}\rangle=\frac{m}{\hbar}\left\langle c, \mathbf{k}\left|\nabla_{\mathbf{k}} H(\mathbf{k})\right| v, \mathbf{k}\right\rangle .
$$

Although Eq. (17) does not consider the intra-atomic part of the momentum matrix element $\stackrel{41.42}{=}$ it is gauge invariant $\stackrel{38.39}{ }$ Its accuracy can be improved by going beyond nearest neighbor interaction or by using non-orthogonal orbitals $\underline{\underline{43}}$ We first calculate the joint densities of states (JDOS) because the variation of the oscillator strength over the Brillouin zone is small. ${ }^{24}$ We assume that light is propagating along the SL growth direction. The JDOS represents the number of states that can undergo energy and $\mathbf{k}$-conserving transitions for photon frequencies between $\omega$ and $\omega+d \omega$. The JDOS associated with Eq. (5) are shown in Fig. [6] for SLs with $n=12$ and $m=4, n=m=8, n=8$ and $m=4$, and $m=n=6$. A $0.05 \mathrm{eV}$ broadening was considered for each electronic energy. The summation over Brillouin zone was replaced by the summation over special points in the Brillouin zone.44.45

Due to band folding the absorption edges for vertical transitions are lowered toward the indirect bandgap of bulk Si. Moreover, the absorption edges are not determined by the 

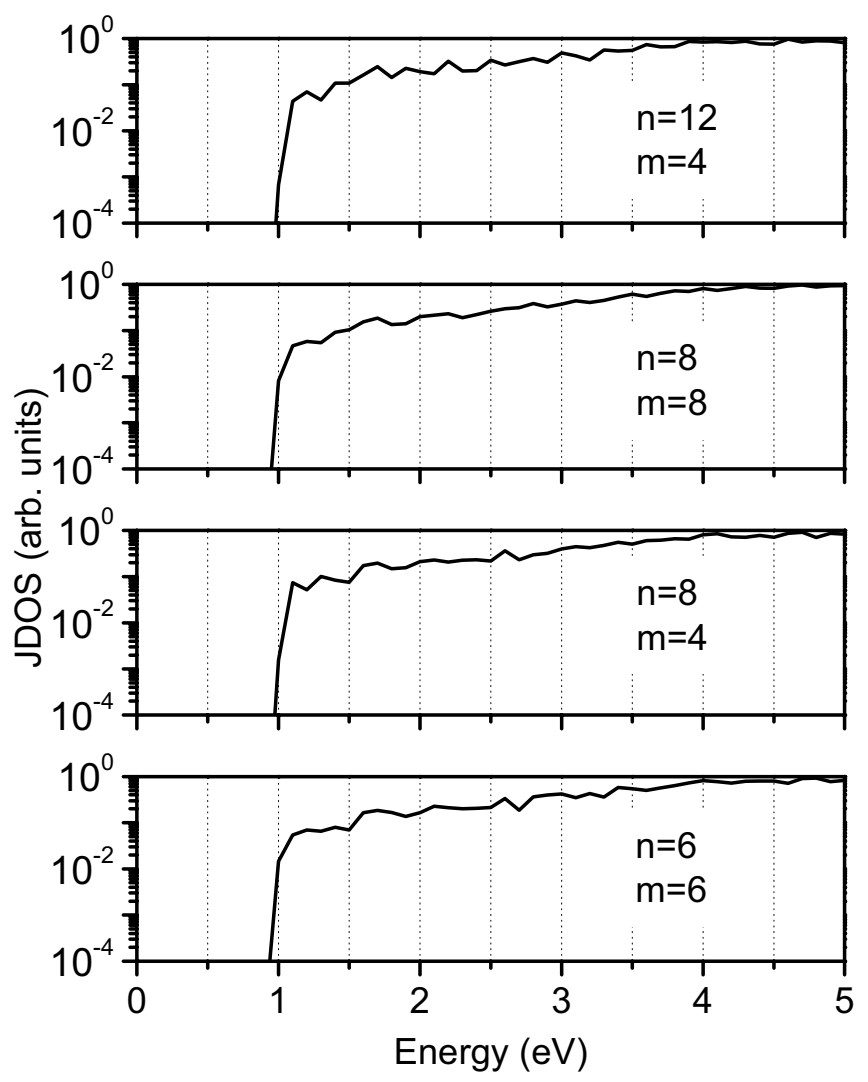

FIG. 6: Joint density of states corresponding to optical transitions of $\left(\mathrm{Si}_{2}\right)_{n} /\left(\mathrm{BeSe}_{0.41} \mathrm{Te}_{0.59}\right)_{m}$ [001] superlattices with $n=12$ and $m=4, n=m=8, n=8$ and $m=6$, and $m=n=6$.

interface subbands but by the energy difference between the lowest empty confined state and highest occupied confined state. Thus the absorption edges for vertical transitions are below the indirect bandgap of Si because the heterostructure is of type II. The curves rise slowly and, with increasing $m(n)$, the absorption edges extend to lower energies. However, the thickness of the $\mathrm{BeSe}_{0.41} \mathrm{Te}_{0.59}$ layers play a greater role than the thickness of Si layers in the variation of the absorption edge. It can be explained by the difference of the electron effective masses in $\mathrm{Si}$ and hole effective masses in $\mathrm{BeSe}_{0.41} \mathrm{Te}_{0.59}$, i.e., the heavy hole effective mass in $\mathrm{BeSe}_{0.41} \mathrm{Te}_{0.59}$ is smaller than the effective mass of heavy electron valley in Si. Similar results were found in the calculations for porous $\mathrm{Si}$ with periodic boundary conditions: ${ }^{42}$ the absorption band edge does not vary much with Si porosity. The strength of the optical absorption is also determined by the oscillator strength, thus we can also check if the transition are allowed or not allowed. We denote by Iv, V1, and V2 the first three top valence subbands, and by Ic, C1, and C2 the first three conduction subbands. We calculated the 


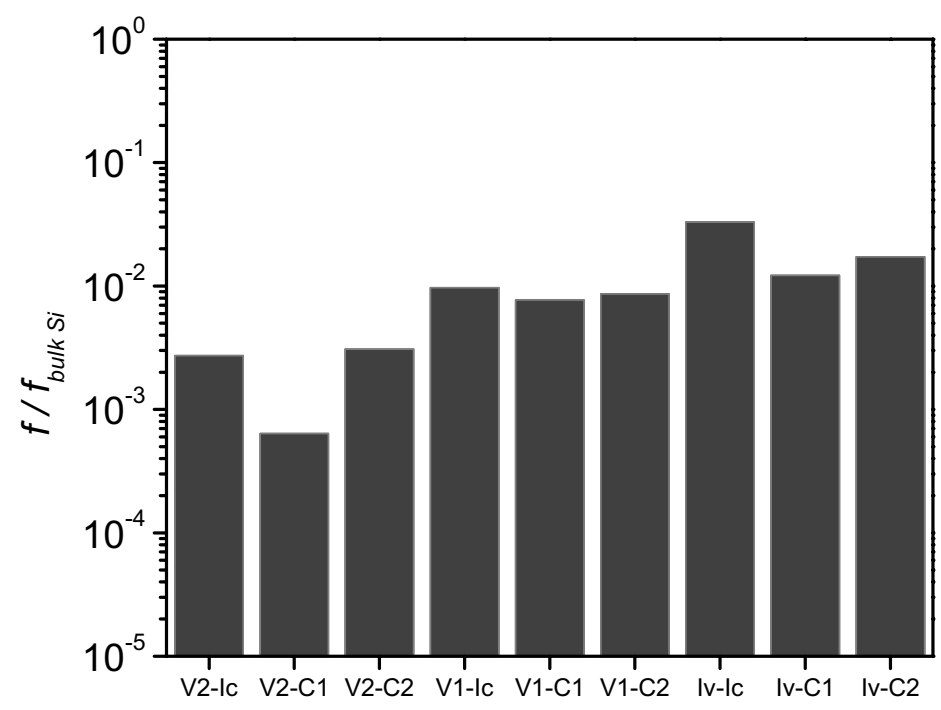

FIG. 7: The oscillator strengths $f$ of several interband transitions relative to the direct transition in bulk $\mathrm{Si}\left(\Gamma_{25}, \quad \rightarrow \Gamma_{15}\right) \quad f_{\text {bulkSi }}$ for the $\left(\mathrm{Si}_{2}\right)_{4} /\left(\mathrm{BeSe}_{0.41} \mathrm{Te}_{0.59}\right)_{4}[001]$ superlattice.

oscillator strengths of several interband transitions relative to the oscillator strength of the direct transition in bulk Si. The results are shown in Fig. 7 for $m=n=4$. The oscillator strengths of interband transitions are at least 10 times smaller than their bulk counterpart and basically range from $10^{-3}$ to $10^{-1}$ relative to $\Gamma_{25}, \quad \rightarrow \quad \Gamma_{15}$ transition in bulk Si. The strongest transitions are those coming from the interface subband and those coming from the first confined hole level to the confined electron levels.

\section{CONCLUSIONS}

We studied the electronic and optical properties of silicon-based heterostructures $\left(\mathrm{Si} / \mathrm{BeSe}_{0.41} \mathrm{Te}_{0.59}\right)$. Based on the fact that significant light absorption occurs for thin uncoupled Si quantum wells we studied the $(\mathrm{Si})_{n} /\left(\mathrm{BeSe}_{0.41} \mathrm{Te}_{0.59}\right)_{m}$ superlattice structures with a semiempirical tight-binding method in the $s p^{3} s^{*}$ nearest neighbor model including spin-orbit interaction. The tight-binding model considered large band bowing in the $\mathrm{BeSe}_{0.41} \mathrm{Te}_{0.59}$ alloy. The heterostructure $\mathrm{Si} / \mathrm{BeSe}_{0.41} \mathrm{Te}_{0.59}$ was found to be of type II. Electronic structure and optical properties were calculated for various superlattice structures. Two interface bands were found to exist in the bandgap of bulk silicon. We found that band folding induces vertical transitions below the indirect bandgap of bulk Si. In addition, calculated oscillator strengths for vertical transitions near the optical band edge show the mixing of the 
zone-center and zone edge states of bulk Si for conduction subbands. Therefore, the transitions are optically allowed and the response of silicon based heterostructures to illumination is enhanced.

\section{Acknowledgments}

This work was supported in part by NASA grant NCC-1-02038 and by the Texas Advanced Technology program under grant No. 003656-0029-2003. The work has been also supported in part by NSERC grants no. 311791-05 and 315160-05. One of the authors (T.S.) wishes to acknowledge generous support in the form of computer resources from the Réseau Québécois de Calcul de Haute Performance.

* Electronic address: titus.sandu@umontreal.ca

† Electronic address: kirk@uta.edu

1 D. J. Paul, Adv. Materials 11, 191 (1999).

2 M. L. Lee and E. A. Fitzgerald, J. Appl. Phys. 97, 011101 (2005).

3 S. S. Iyer and Y. H. Xie, Science 260, 40 (1993).

4 R. Tsu, Nature 364, 338 (1993).

5 A. G. Cullis, L. T. Canham, and P. D. J. Calcott, J. Appl. Phys. 82, 909 (1997).

6 F. A. DAvitaya, L. Vervoort, S. Ossicini, A.Fasolino, and F. Bernardini, Europhys. Lett. 31, 25 (1995).

7 D. Lockwood, Z. Lu, and J.-M. Baribeau, Phys. Rev. Lett. 76, 539 (1996).

8 A. Nassiopoulos, S. Grigoropoulos, D. Papadimitiriu, and E. Gogolides, Phys. Stat. Sol. (b) 190, 91 (1996).

9 Y. Kanemitsu, H. Uto, Y. Masumoto, T. Matsumoto, T. Futagi, and H. Mimura, Phys. Rev. B 48, 2827 (1993).

10 Y. Niiyama and M. Watanabe, Semicond. Sci. Technol. 20, 1187 (2005).

11 K. Kishino and I. Nomura, Phys. Stat. Sol. (c) 1, 1477 (2004).

12 K. Clark, E. Maldonado, P. Barrios, G. F. Spencer, R. T. Bate, and W. P. Kirk, J. Appl. Phys. 88, 7201 (2000). 
13 W. P. Kirk, K. Clark, E. Maldonado, N. Basit, R. T. Bate, and G. F. Spencer, Supperlatt. Microstruct. 28, 377 (2000).

14 P. Vogl, H. P. Hjalmarson, and J. D. Dow, J. Phys. Chem. Solids 44, 365 (1983).

15 J. C. Slater and G. F. Koster, Phys. Rev. 94, 1498 (1954).

16 R. Jones and O. Gunnarsson, Rev. Mod. Phys. 61, 689 (1989).

17 W. Aulbur, L. Jonsson, and J. W. Wilkins, in Solid State Physics, edited by H. Ehrenreich and F. Spaepen (Academic Press, San Diego San Francisco New York Boston London Sydney Tokyo, 2000), vol. 54, p. 1.

18 T. Sandu and W. P. Kirk, Phys. Rev. B 72, 073204 (2005).

19 W. A. Harrison, Electronic Structure and the Properties of Solids (Freeman, San Francisco, 1980).

20 D. J. Chadi, Phys. Rev. B 16, 790 (1977).

21 A. Fleszar and W. Hanke, Phys. Rev. B 62, 2466 (2000).

22 M. S. Hybertsen, Phys. Rev. Lett. 72, 1514 (1994).

23 M. Gell, D. Nino, M. Jaros, and D. C. Herbert, Phys. Rev. B 34, 2416 (1986).

24 J. N. Schulman and Y. C. Chang, Phys. Rev. B 31, 2056 (1985).

25 G. Klimeck, R. C. Bowen, T. B. Boykin, C. Salazar-Lazaro, T. A. Cwik, and A. Stoica, Supperlatt. Microstruct. 27, 77 (2000).

26 W. A. Harrison, E. A. Kraut, J. R. Waldrop, and R. W. Grant, Phys. Rev. B 18, 4402 (1978).

27 H. Kroemer, J. Cryst. Growth 81, 193 (1987).

28 R. Eppenga, Phys. Rev. B 40, 10402 (1989).

29 F. Della Sala, A. Di Carlo, P. Lugli, F. Bernardini, V. Fiorentini, R. Scholz, and J. M. Jancu, Appl. Phys. Lett. 74, 2002 (1999).

30 A. Bonfiglio, M. Lomascolo, G. Traetta, R. Cingolani, A. Di Carlo, F. Della Sala, P. Lugli, A. Botchkarev, and H. Morkoc, J. Appl. Phys. 87, 2289 (2000).

31 T. Saito and T. Ikoma, Phys. Rev. B 45, 1762 (1992).

32 A. Laref, H. Aourag, B. Belgoumene, and A. Tadjer, J. Appl. Phys. 94, 5027 (2003).

33 A. Laref, S. Laref, B. Belgoumene, B. Bouhafs, A. Tadjer, and H. Aourag, J. Appl. Phys. 99, 043702 (2006).

34 E. G. Wang and C. S. Ting, Phys. Rev. B 51, 9791 (1995).

35 E. G. Wang, Appl. Surf. Sci. 105/105, 626 (1996). 
36 S. M. Sze, Physics of Semiconductor Devices (John Wiley and Sons, New York-ChichesterBrisbane-Toronto, 1980), 2nd ed.

37 T. B. Boykin, Phys. Rev. B 52, 16317 (1995).

38 M. Graf and P. Vogl, Phys. Rev. B 51, 4940 (1995).

39 T. B. Boykin and P. Vogl, Phys. Rev. B 65, 035202 (2002).

40 T. B. Boykin, R. C. Bowen, and G. Klimeck, Phys. Rev. B 63, 245314 (2001).

41 T. G. Pedersen, K. Pedersen, and T. B. Kriestensen, Phys. Rev. B 63, 201101(R) (2001).

42 M. Cruz, M. R. Beltran, C. Wang, J. Taguena-Martinez, and Y. G. Rubo, Phys. Rev. B 59, 15381 (1999).

43 T. Sandu, Phys. Rev. B 72, 125105 (2005).

44 D. J. Chadi and M. L. Cohen, Phys. Rev. B 8, 5747 (1973).

45 S. Y. Ren and J. D. Dow, Phys. Rev. B 38, 1999 (1988). 